\title{
A Resource-Constrained Optimal Control Model for Crackdown on Illicit Drug Markets
}

\author{
A. Baveja ${ }^{1}$, G. Feichtinger ${ }^{2}$, R.F. Hartl ${ }^{3}$, J.L. Haunschmied ${ }^{2}$, P.M. Kort ${ }^{4}$
}

\begin{abstract}
In this paper we present a budget-constrained optimal control model aimed at finding the optimal enforcement profile for a street-level, illicit drug crackdown operation. The objective is defined as minimizing the number of dealers dealing at the end of the crackdown operation, using this as a surrogate measure of residual criminal activity. Analytical results show that optimal enforcement policy will invariably use the budget resources completely. Numerical analysis using realistic estimates of parameters shows that crackdowns normally lead to significant results within a matter of a week, and if they do not, it is likely that they will be offering very limited success even if pursued for a much longer duration. We also show that a ramp-up enforcement policy will be most effective in collapsing a drug market if the drug dealers are risk-seeking, and the policy of using maximum enforcement as early as possible is usually optimal in the case when the dealers are risk averse or risk neutral. The work then goes on to argue that the underlying model has some general characteristics that are both reasonable and intuitive, allowing possible applications in focussed, local enforcement operations on other similar illegal activities.
\end{abstract}

JEL-codes: C61, K42

Keywords: crackdown enforcement, illicit drug markets, optimal control

${ }^{1}$ School of Business, Rutgers University, Camden, NJ 08102, USA; baveja @ crab.rutgers.edu.

${ }^{2}$ Institute of Econometrics, OR and Systems theory, University of Technology, Argentinierstr. 8, A-1040 Vienna, Austria; or@e119ws1.tuwien.ac.at; haunschm@e119ws1.tuwien.ac.at.

${ }^{3}$ Institute of Management, University of Vienna, Brünnerstr. 72, A-1210 Vienna, Austria; richard.hartl@univie.ac.at

${ }^{4}$ Department of Econometrics and CentER, Tilburg University, PO Box 90153, NL-5000 LE Tilburg, Netherland; Kort@kub.nl. 


\title{
A Resource-Constrained Optimal Control Model for Crackdown on Illicit Drug Markets
}

\author{
A. Baveja, G. Feichtinger, R.F. Hartl, J.L. Haunschmied, P.M. Kort
}

\section{Contents}

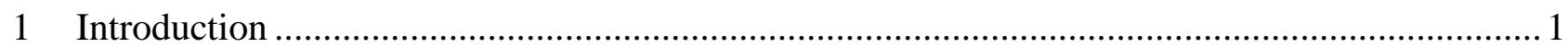

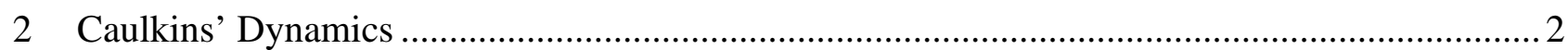

3 Budget-Constrained Crackdown Optimization Model............................................................ 5

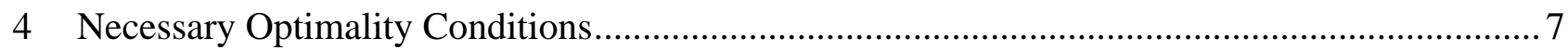

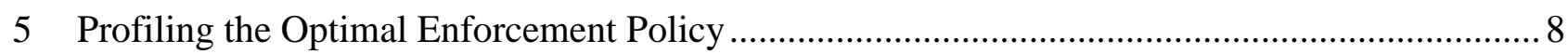

5.1 Risk-Seeking Dealers - Decreasing Returns to Scale of Enforcement ................................. 8

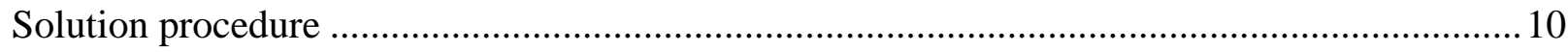

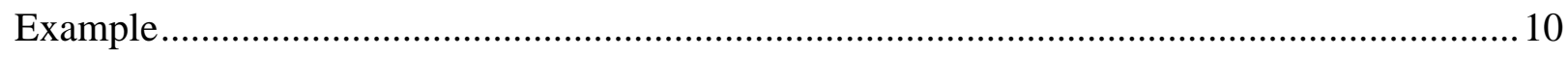

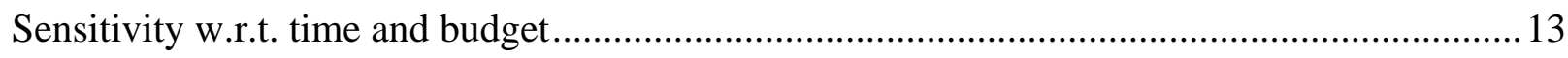

5.2 Non-Risk-Seeking Dealers - Constant and Increasing Returns to Scale of Enforcement ....14

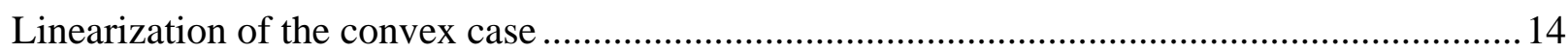

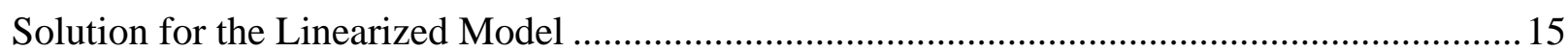

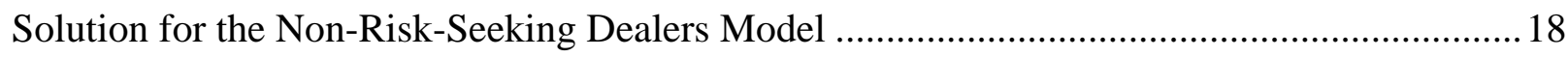

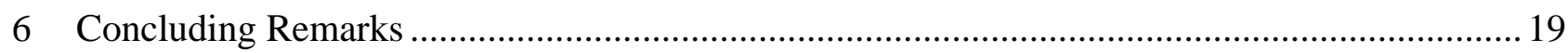

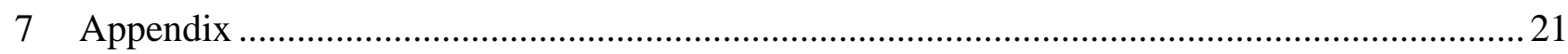

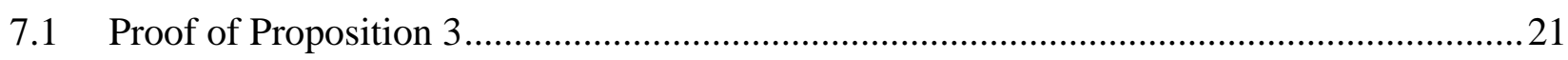

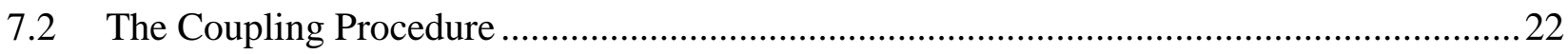

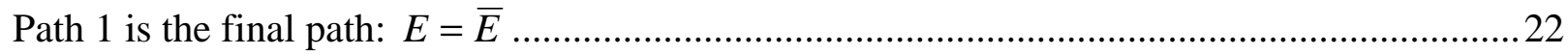

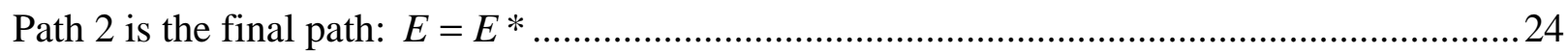

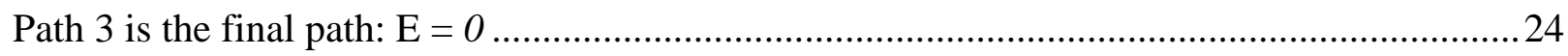

References: 


\section{Introduction}

Illicit drugs continue to impose a significant cost on our society. Today, it is perhaps one of the greatest challenges confronting the law enforcement officials, social activists, and citizens. Increasing taxpayer resources are being spent to confront the illicit drug trade and its associated crimes. The choice of most effective enforcement strategies i.e. local vs. border control, enforcement vs. treatment [Rydell, Caulkins and Everingham 1996], and education, while important questions, is not the focus of this paper.

Despite differences in emphasis and paradigms of the political administrations, resources spent on local enforcement continue to grow. Many observers, politicians, and researchers attribute the recent reduction of crime in the United States to greater and more effective police presence on the streets. With this viewpoint gaining ground, especially with ongoing positive results, increased street/local enforcement may be a trend that we will continue to see. However, the bad news is that there is mounting pressure on law enforcement agencies to demonstrate results especially in "problem" neighborhoods. Achieving these results may require use of decision-aids that enable identification and evaluation of innovative, efficacious enforcement strategies to "recover" highcrime neighborhoods. The Drug Market Analysis (DMA) study in Jersey City, NJ [NIJ 1996] showed a strong impact of novel policing strategy in reducing crime indicators and calls for emergency service, underscoring the importance of pursuing tactics that are innovative.

One strategy that continues to be used, often as a first step to revitalization of neighborhoods, is a drug crackdown - i.e., concentration of enforcement resources in a geographic area for a limited time [Worden et al. 1992] or for focussing on specific types of crime [NIJ 1996]. However, for the purpose of this paper we will use the restricted definition of focussing on a geographic area. The limited studies done thus far on crackdowns can be categorized into two groups - evaluating the impact of a crackdown operation [NIJ 1996, Barnett 1988, Caulkins et al. 1993, Forero 1990, Kleiman 1988, Zimmer 1987], and modeling the effect of crackdown on illicit drug markets [Caulkins 1990, Caulkins 1993, Baveja et al. 1993, Baveja et al. 1997, Kort et al. 1998]. This paper extends the work in the second category by modeling the impact of such crackdowns based on Caulkins' utility-based, dynamic model for predicting the flow of drug dealers into (out of) a drug market as a function of dealers' profit and the enforcement risk.

Caulkins' model lends itself to optimal control literature especially to address the question of optimal time trajectory of enforcement during a crackdown operation. Prior work by Baveja et al. [1997] and Kort et al. [1998] used the objective of minimizing the enforcement resources (objective function) with a constraint of collapsing the drug market. In reality, however, law enforcement budget may be fixed and is better modeled via a constraint in an optimization model. Furthermore, 
some policy experts [Kleiman 1992] argue that drug policy should focus on how best to "manage" the problem and the slogan "war on drugs" is misplaced. Consistent with this philosophy, the current work focuses attention on reducing the size of a drug market to as small as possible subject to the restriction of the law enforcement budget and a pre-specified time for the crackdown operation. Thus this research is dealing with a more realistic optimization scenario to the important problem of illicit drug crackdowns, consistent with the viewpoint put forth by some leading drug policy researchers.

The rest of the paper is organized as follows. In the next section we will briefly review Caulkins' crackdown model along with some of its underlying dynamics. Section 3 will formulate the optimal control model investigated in this paper following which we will present generalized analytical conditions for optimality. Section 5 will look at application of these conditions to determine the optimal enforcement policies utilizing example data. Finally we discuss the implications of this work along with the inherent limitations and directions for future work.

\section{Caulkins' Dynamics}

Caulkins (1993) considers a province or larger city with a large number of identical dealers that are spread over a large number of drug markets.

Each day every dealer goes to the market that offers the best opportunity. This is not only determined by the expected dollar profit but also by non-monetary factors such as risks of enforcement, threats of violence by other market participants, etc. In the equilibrium, this "generalized profit" per dealer should be the same in all markets.

Consider the following notation:

$\omega_{0}=$ the equilibrium level of the generalized profit or the reservation wage

If in a certain market the generalized profit exceeds $\omega_{0}$, then new dealers will enter. On the other hand, if the generalized profit in a market is below $\omega_{0}$, then dealers will leave.

Further, if:

$N(t)=$ number of dealers at time $t$ in a certain market under consideration

$\pi=$ generalized profit per unit of sales in this market $(\pi>0$ and constant $)$

assuming that sales depend on the number of dealers in the following way:

$\alpha N^{\beta}=$ number of sales per day provided that there are $N$ dealers, where

$\alpha>0$ and $\beta \in[0,1]$ are constants; 
then the generalized profit due to sales per dealer is $\frac{\pi \alpha N^{\beta}}{N}$.

The parameter $\beta$ is a crucial one: the higher $\beta$ the more total sales of the market depend on the number of dealers. If $\beta=1$ the drugs market is a sellers' market where each dealer brings his own customers with him. If $\beta=0$ the total number of customers in the market is fixed (buyers' market) and thus independent of the number of dealers. In the latter case it is less attractive for a dealer to enter such a market if the number of dealers active in this market is already large.

Also, if

$E(t)=$ enforcement effort associated with the crackdown at time $t$, and

$\gamma=$ parameter associated with per dealer cost of enforcement effort $(\gamma>0$ and constant),

then the generalized net profit per dealer incorporating risk due to enforcement is $\frac{\pi \alpha N^{\beta}}{N}-\left(\frac{E}{N}\right)^{\gamma}$. Clearly, the effect of crackdown efforts completely depends on the value of $\gamma$. It is assumed that the burden of enforcement is equally shared among the dealers. This implies that, given the enforcement effort more enforcement pressure is felt by an individual dealer in case there are only a few dealers active in the market. Caulkins calls the case $\gamma<1$ that of risk seeking dealers, while $\gamma$ $=1$ and $\gamma>1$ characterize risk neutral and risk averse dealers, respectively.

The well known Caulkins' dynamics, defined for $N>0$, says that

$$
\dot{N}=c_{1}\left[\pi \alpha N^{\beta-1}-\left(\frac{E}{N}\right)^{\gamma}-\omega_{0}\right]
$$

where

$c_{1}=$ the speed of adjustment parameter $\left(c_{1}>0\right.$ and constant $)$.

It is reasonable to assume that the parameters satisfy

$$
\pi \alpha>\omega_{0},
$$

since otherwise the market under consideration is not attractive and the drug market will disappear even without a crackdown, obviously an unrealistic case. From (1) it is clear that, whether the value of $\gamma$ exceeds one or not will determine if there are decreasing or increasing returns to scale of enforcement activities.

Before we formulate the optimization problem based on equation (1), let us discuss the dynamics that governs the dealer population a little further, as it will help the analysis in the following sections. 
For $N>0$, one can write (1) also as

$$
\dot{N}=\frac{c_{1}}{N^{\gamma}}\left(\pi \alpha N^{\gamma+\beta-1}-E^{\gamma}-\omega_{0} N^{\gamma}\right) \text {. }
$$

From this expression it can be obtained that a market collapse (i.e. $N=0$ ) is not possible if $\gamma+\beta<1$, since in this case the first term within brackets goes to infinity when $N$ approaches zero. If we assume for the moment that $\gamma+\beta-1>0$, the situation can be illustrated in Fig. 1 .

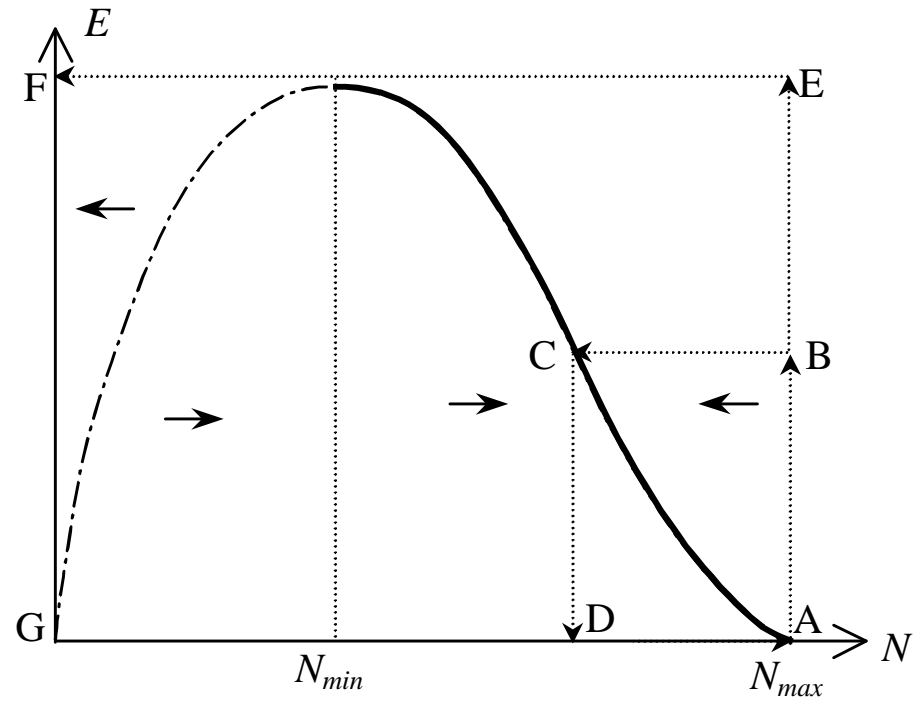

Figure 1. The Caulkins' dynamics. The bold line denotes stable states. "Pushing the balloon a little" with low enforcement: ABCDA; the balloon is popped with high enforcement: AEFG.

Let us first compute the long-run equilibrium without enforcement, by equating the part between parentheses in (3) to zero. This yields:

$$
N_{\max }=\left(\frac{\pi \alpha}{\omega_{0}}\right)^{\frac{1}{1-\beta}} .
$$

Clearly $N_{\max }$ can never be exceeded when starting below this value. Further, different steady state levels of $N$ can be determined as a function of the enforcement level. The highest enforcement level associated with a positive steady state level of dealers, leads to the following equilibrium level for the number of dealers:

$$
N_{\min }=\left(1-\frac{1-\beta}{\gamma}\right)^{\frac{1}{1-\beta}} N_{\max }=\left(\frac{\gamma+\beta-1}{\gamma}\right)^{\frac{1}{1-\beta}}\left(\frac{\pi \alpha}{\omega_{0}}\right)^{\frac{1}{1-\beta}},
$$

where according to (3) the attractiveness of the market to new dealers is particularly high. This is reflected by the fact that a very high enforcement level is needed to keep the number of dealers constant. It is obvious that $N_{\min }>0$ is only defined for $\gamma+\beta>1$. 
In order to complete the discussion of the shape of the curve in Fig.1, we note that it has an inflection point between $N_{\min }$ and $N_{\max }$ [see p.182 of Kort et al. 1998]. However, the exact shape is not really important and it suffices to know that it is bell shaped.

If starting from a situation (point $\mathrm{A}$ in Fig. 1) with a high number of dealers, e.g. $N_{\max }$, then exerting a low enforcement effort means just "pushing the balloon a little" since the movement according to (3) is from B to the new equilibrium level C. As soon as the crackdown is over, enforcement jumps to zero and the solution jumps to point $\mathrm{D}$ from where the previous equilibrium (point A) is again approached.

The "balloon is popped" only with high enforcement effort, since then the movement is from point $\mathrm{E}$ to point $\mathrm{F}$, where the dealer population is extinct. Note that for $N=0$ the Caulkins' dynamics (1) or (3) is not defined, but we make the reasonable assumption that in such a situation the dealer population would stay at zero level.

\section{Budget-Constrained Crackdown Optimization Model}

Past optimization work based on Caulkins' dynamic equation has focused on minimizing the enforcement resources subject to the constraint of collapsing the drug market. However, the total enforcement budget available is rarely under the direct control of the law-enforcement decisionmaker and often is a pre-specified resource. Typically, the objective of a crackdown operation is to minimize the street drug dealing as much as possible keeping within the budgetary constraint. Mathematically, this is a new, uninvestigated, relevant problem requiring challenging optimization solution methodologies. To specify the model, we need to define the following additional parameters:

$$
\begin{aligned}
& B=\text { budget available for the crackdown }(B>0 \text { and constant }), \\
& T=\text { horizon date }(T>0 \text { and constant }) \\
& r=\text { discount rate }(r>0 \text { and constant }) .
\end{aligned}
$$

The Budget-Constrained Crackdown Optimization Model (BCOM) can be written via the following optimal control formulation:

$$
\text { Minimize } e^{-r T} N(T) \text {, }
$$

subject to

$$
\dot{N}=c_{1}\left[\pi \alpha N^{\beta-1}-\left(\frac{E}{N}\right)^{\gamma}-\omega_{0}\right], \quad N(0)=N_{0},
$$




$$
\int_{0}^{T} E(t) d t \leq B .
$$

Alternatively, the objective could be extended by adding the discounted integral of the number of dealers throughout the crackdown period. Since such a crackdown period is usually short (a week or so), we choose to ignore the impact of number of dealers during the crackdown period in the objective function.

Note that even though we have used $N(T)$ in the objective function, it can be replaced by a function, $f(N(T))$, with $f^{\prime}>0$ for all $N(T)$, without changing the solution of the optimal enforcement profile. This is true, because $f(N(T))$ is minimized by minimizing $N(T)$, since $f^{\prime}>0$. The model also emphasizes that if the drug market is not collapsed at the end of the crackdown operation (i.e., $N(T)>0$ ) and no post-crackdown enforcement/recovery programs are in place, the gains achieved maybe quickly lost with the market bouncing back to a level of $N_{\max }$. Therefore, in investigating this model we assume that a crackdown operation is followed up by a phase 2 which targets improving lighting/housing/facilities and bringing businesses/residents back into the area. It is reasonable to assume that the cost of such a Phase 2 project is increasing in the number of dealers remaining, $N(T)$. Note that this model is still valid even if the cost of the post-crackdown operation is nonlinear in $N(T)$, provided the reasonable property that the function is increasing in $N(T)$ holds.

The isoperimetric constraint (8) can as usual be transformed into a terminal value constraint by introducing a new state variable:

$D(t)=$ money spent on crackdown enforcement on $[0, t)$.

Incorporating this variable, we restate the formulation as:

$$
\text { Minimize } e^{-r T} N(T)
$$

subject to

$$
\begin{gathered}
\dot{N}=c_{1}\left[\pi \alpha N^{\beta-1}-\left(\frac{E}{N}\right)^{\gamma}-\omega_{0}\right], N(0)=N_{0}, \\
\dot{D}=E, \quad D(0)=0 \\
D(T) \leq B .
\end{gathered}
$$

with two states, $N$ and $D$, and one control, $E$.

The $\exp (-r T)$ term in the objective function (6) plays no role. It is included because it does play a role in subsequent free end state versions of the problem. For a fixed $T$, discounting does not change the optimal solution as we will see e.g. from (17) in Section 5.

Next, we investigate necessary conditions for the optimal trajectory. 


\section{Necessary Optimality Conditions}

In this section we employ optimal control theory to establish some analytical results that will help us in understanding the dynamics of the optimal solution. We first formulate the Hamiltonian (see, e.g., Feichtinger and Hartl (1986)):

$$
H=\lambda_{1} c_{1}\left[\pi \alpha N^{\beta-1}-\left(\frac{E}{N}\right)^{\gamma}-\omega_{0}\right]+\lambda_{2} E,
$$

and obtain the necessary optimality conditions:

\section{$E$ maximizes $H$.}

Since $\lambda_{1}, \lambda_{2}$ are shadow prices of the undesirable states, "number of dealers" and "budget already used", respectively, it is reasonable to consider $\lambda_{1} \leq 0, \lambda_{2}<0$. Below (15) we show that these inequalities indeed hold. If we assume that $\gamma<1$, i.e. dealers are risk seeking (the case of $\gamma \geq 1$ will be considered separately in Sections 6 and 7), we get

$$
H_{E}=-\lambda_{1} c_{1} \gamma E^{\gamma-1} N^{-\gamma}+\lambda_{2}=0 .
$$

Furthermore we have:

$$
\begin{aligned}
& \dot{\lambda}_{1}=r \lambda_{1}-H_{N}=\lambda_{1}\left[-(\beta-1) c_{1} \pi \alpha N^{\beta-2}-\gamma c_{1} E^{\gamma} N^{-\gamma-1}+r\right\rfloor \text {, } \\
& \dot{\lambda}_{2}=r \lambda_{2}-H_{D}=r \lambda_{2} \text {, } \\
& \lambda_{1}(T)=-1 \text {, } \\
& \lambda_{2}(T)=-\alpha, \quad \alpha \geq 0, \quad \alpha(B-D(T))=0 .
\end{aligned}
$$

Note that (15) comes from constraint (9b) and implies $\lambda_{2}(T) \leq 0$. Hence, by (13), $\lambda_{2}(t) \leq 0$ for all $t$. From (11), this in turn implies $\lambda_{1}(t) \leq 0$ for all $t$.

Let us now make the following observation:

Proposition 1. If the budget B is finite, it is always optimal to spend the whole budget:

$$
D(T)=B .
$$

Proof: Assume on the contrary that $D(T)<B$ which, by (15), implies $\alpha=0$ and $\lambda_{2}(t)=0$ for all $t$, because of (13). Thus, the optimal solution, by (11), would be $E=\infty$ for all $t$ where $\lambda_{1}<0$, which at least holds on a final time interval, say $(\tau, \mathrm{T}]$. However, by this solution constraint (8) is violated.

The above proposition implies that an optimal crackdown enforcement strategy necessarily exhausts the budget resources completely. This is intuitively appealing and practically significant since it indirectly implies that "left-over" resources can always be beneficially utilized and never 
does it make sense to leave resources unused. This result is consistent with the essence of the argument for the crackdown operation that a concentrated use of resources is beneficial.

The proof of Proposition 1 also shows that, in fact, $\lambda_{2}<0$, since otherwise $\alpha=0$ would imply $E=\infty$ for all $\mathrm{t}$. On the other hand, $\lambda_{1}<0$ is clear since otherwise the Hamiltonian maximizing condition implies $E=0$ which again gives the worst solution possible.

In the next section we discuss the actual optimal policies under the conditions of $\gamma<1, \gamma=1$ and $\gamma>1$.

\section{Profiling the Optimal Enforcement Policy}

To understand the characteristics of the optimal enforcement policy for the BCOM, we consider the following three exhaustive cases - risk seeking dealer $(\gamma<1)$, risk neutral dealer $(\gamma=1)$, and risk averse dealer $(\gamma>1)$.

\subsection{Risk-Seeking Dealers - Decreasing Returns to Scale of Enforcement}

In this case $\gamma<1$ and the Hamiltonian is strictly concave in $E$. Therefore the optimal $E$ is continuous over time and follows the differential equation

$$
\dot{E}=\frac{c_{1} E}{(1-\gamma) N}\left[-\pi \alpha(\gamma+\beta-1) N^{\beta-1}+\gamma \omega_{0}\right] .
$$

To understand the implications of this equation, we present the following proposition.

Proposition 2. For the monotonicity of enforcement effort we have to consider two cases:

a) when $\gamma+\beta \leq 1$ then $\dot{E}>0$ is always optimal (ramp up enforcement);

b) when $\gamma+\beta>1$ then $\dot{E}\left\{\begin{array}{l}> \\ <\end{array}\right\} 0$ if $N\left\{\begin{array}{l}> \\ <\end{array}\right\} N_{\min }$ with $N_{\text {min }}$ from (5).

We can now analyze the phase diagram for $\gamma+\beta>1$ and $\beta<1$, which is depicted in Figure 2 .

Proposition $2 \mathrm{~b}$ is reflected in the position of the vertical part of the $\dot{E}=0$ isocline which is exactly at $N=N_{\min }$. This implies that enforcement decreases for $N<N_{\min }$ while E increases for $N>N_{\text {min }}$. The $\dot{N}=0$ isocline is the same as in Figure 1 . 


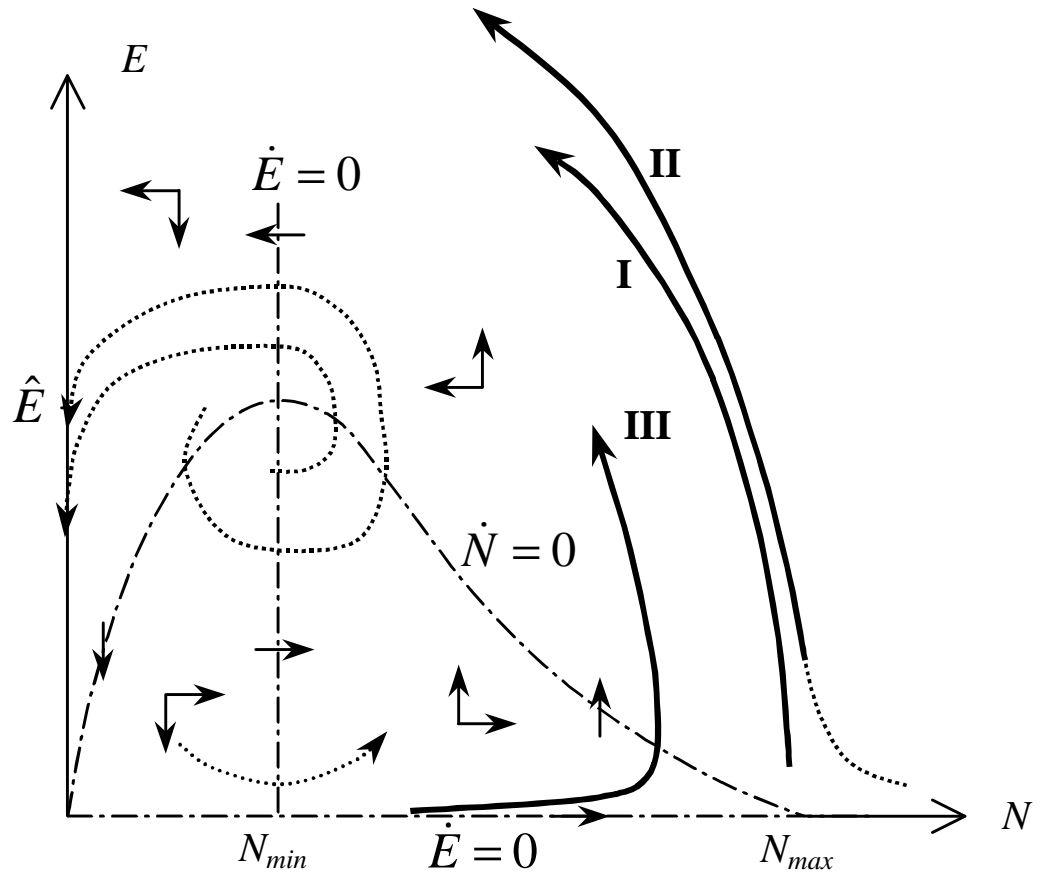

Figure 2. The phase diagram for $\gamma+\beta>1$ and $\beta<1$.

The equilibrium $\left(N_{\min }, \hat{E}\right)$ with $\hat{E}=N_{\min }\left(\pi \alpha N^{\beta-1}-w_{0}\right)^{1 / \gamma}$ is unstable, since the Jacobian determinant is (note that $\partial \dot{E} / \partial E=0$ in this equilibrium) (see Feichtinger and Hartl (1986)):

$$
\frac{\pi \alpha \gamma(1-\beta)(\gamma+\beta-1) c_{1}^{2}}{1-\gamma} N_{\min }^{\beta-\gamma-3} \hat{E}^{\gamma}>0 .
$$

The other equilibrium $\left(N_{\max }, 0\right)$ is a saddle point, since the Jacobian determinant becomes

$$
-\frac{\pi \alpha(1-\beta) c_{1}^{2}}{1-\gamma} N_{\max }^{\beta-3}\left[\gamma \omega_{0}-\pi \alpha(\gamma+\beta-1) N^{\beta-1}\right]<0,
$$

when approaching this equilibrium. Note that while $\partial \dot{N} / \partial E=-\gamma c_{1} E^{\gamma-1} N^{-\gamma}$ tends to minus infinity, the product $(\partial \dot{N} / \partial E)(\partial \dot{E} / \partial N)$ tends to zero when approaching this equilibrium.

The proposition is best understood in the context of the highest attractive state of the market to a dealer, $N_{\min }$; see (5). From Figure 1 it is clear that if $N>N_{\min }$, and as $N$ decreases (caused by the current crackdown effort), more enforcement intensity will become necessary to reduce the number of dealers. In other words, the market appears more attractive to a dealer therefore requiring an increased enforcement-intensity as a function of time - $\dot{E}>0-$ as the proposition suggests. This argument also holds for the first part of the proposition where $N_{\min }\langle 0$ ensuring that $N\rangle N_{\min }$.

On the other hand, when $N<N_{\text {min }}$, the enforcement pressure felt by an individual dealer is large making it exceedingly difficult for dealers to deal. Therefore, a reduced enforcement effort may be acceptable - $\dot{E}<0$ - as suggested in the second part of the proposition. 


\section{Solution procedure}

Since the transversality conditions do not give the values of $E(T)$ and $N(T)$, it is not immediately clear which of the various solution candidates (extremals of the canonical system) represents the optimal solution. The answer is, that for a given $N(0)$, the corresponding $E(0)$ has to be chosen such that the solution of system (7) and (17) satisfies the budget constraint (16).

For a small budget and $N=N_{\max }$ (the equilibrium for $E=0$ ) we have trajectory I in Fig. 2. For a moderate budget, starting from $N=N_{\max }$, we have trajectory II.

If $N$ is very small and $B$ is also very small, then trajectory III could occur where $N$ increases initially. At first sight, this is not a realistic situation, since at the beginning of a crackdown $N$ is usually large. However, it could occur if in a previous period a crackdown was carried out which reduced the number of dealers but not enough to collapse the market. Trajectory III signals a point of caution for enforcement personnel. The lesson here is that "a small drug market does not automatically justify a small crackdown budget as it may be counterproductive".

Next, the solution procedure ${ }^{5}$ is illustrated via an example.

\section{Example}

To illustrate this solution methodology we choose the following parameter values from illicit drug sales estimates in Washington, D.C. [Baveja et al. (1997)]:

$N_{0}=100, \omega_{0}=50$ (typical reservation wage per dealer per day based on a monthly average of $\$ 1046) . \beta=0.5, \pi \alpha=500$ (estimated based on $N_{0}=N_{\max }$ ).

However, different from Baveja et al. (1997) we initially choose $\gamma=0.6$. Baveja's original choice of $\gamma>1$ leads to a convex problem which is dealt with in the next section. Note that $\gamma+\beta>1$ and $\beta<1$ so that Figure 2 applies.

For these values we obtain:

$$
N_{\min } \approx 2.78, N_{\max }=100,
$$

and the enforcement effort in the unstable equilibrium $\hat{E} \approx \$ 185000$. Proposition 2 implies that the enforcement effort is increasing over time except when $N<2.78$ in which case it is decreasing. Now for a typical crackdown time interval of $T=7$ days we have plotted the above mentioned diagram $E(0)$ vs. $D(T)$ for different initial budgets $B$ :

\footnotetext{
${ }^{5}$ For a given $N(0)$ the amount of budget $D(T)$ used up by the terminal time $T$ according to (9a) will be an increasing function of $E(0)$. Thus we have to find an $E(0)$ value such that $D(T)=B$. This is illustrated next via an example.
} 


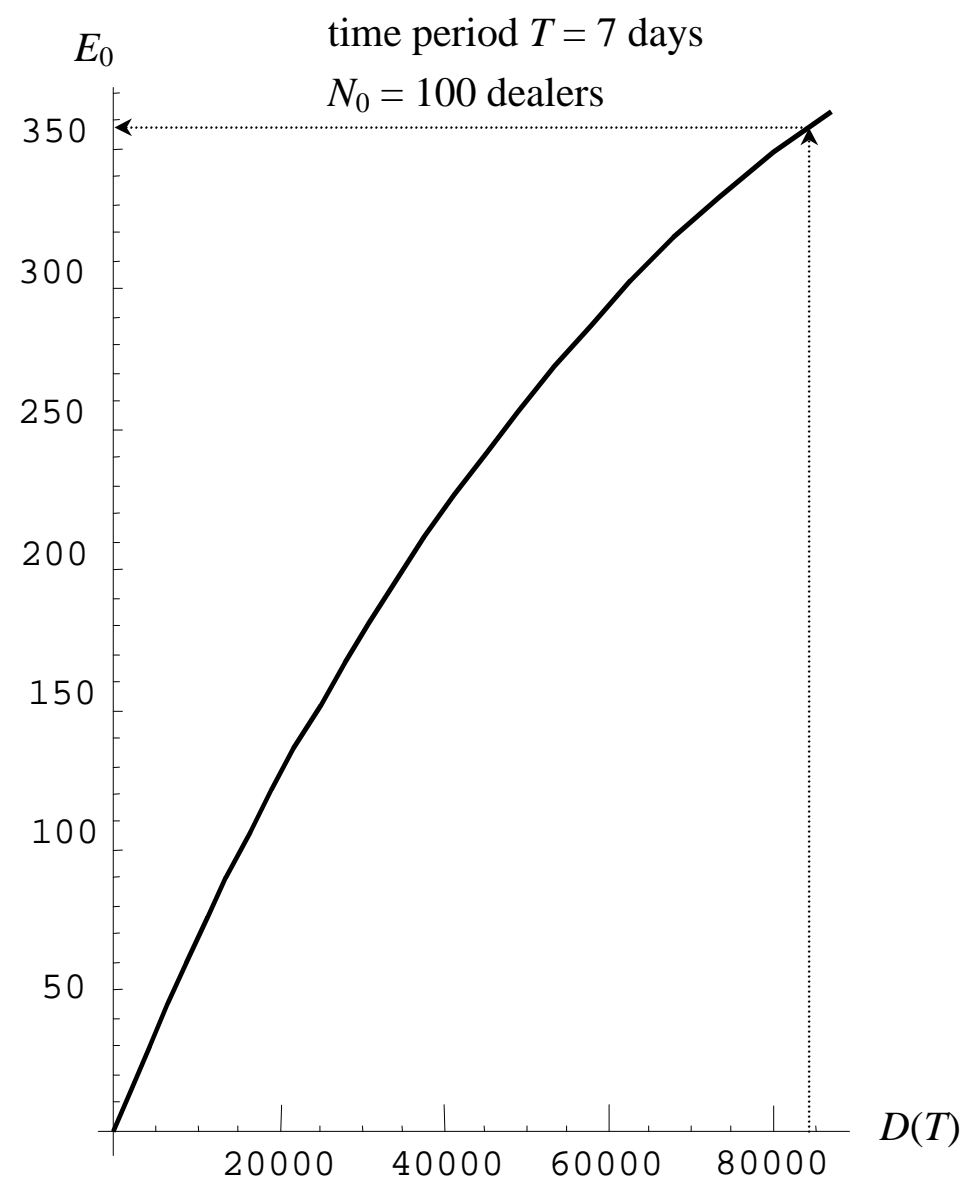

Figure 3. Budget used, $D(T)$, within $T=7$ days, starting from different initial enforcement levels $E(0)$ and following the canonical system.

Assuming an average weekly salary of $\$ 1200$ per enforcement officer, the personnel cost of 20 officers would be $\$ 24,000 /$ week. For a round-the-clock three eight-hour shift schedule, this would imply a cost of $\$ 72,000$ for a week. With some additional overheads we estimated the budget of a weeklong crackdown operation to be $\$ 84,000$. For a given budget, in our case for $B=\$ 84,000$, we can obtain that the initial enforcement should be about $\$ 369$ per day. For a given budget, e.g. again for $B=\$ 84,000$, we can also look at the enforcement profile, i.e. at the time path of $E(t)$ (Figure 4).

From the figure it is clear that we have obtained an extreme ramp-up enforcement profile. This is consistent with the claim of Proposition 2 where $\gamma+\beta>1$ and $N>N_{\min }$, implying $\dot{E}>0$. Intuitively, enforcement's effect is most felt when $N$ decreases (i.e. later in the crackdown operation) resulting in the risk factor $-(E / N)^{\gamma}$ to increase making dealers leave the market. Practically, the ramp-up strategy has a psychological benefit - the maximum impact of the crackdown will be felt towards the end of the crackdown operation, an important morale booster for law enforcement agencies, which may convince residents, activists and city officials to commit additional ongoing resources for follow-up neighborhood revitalization efforts. 


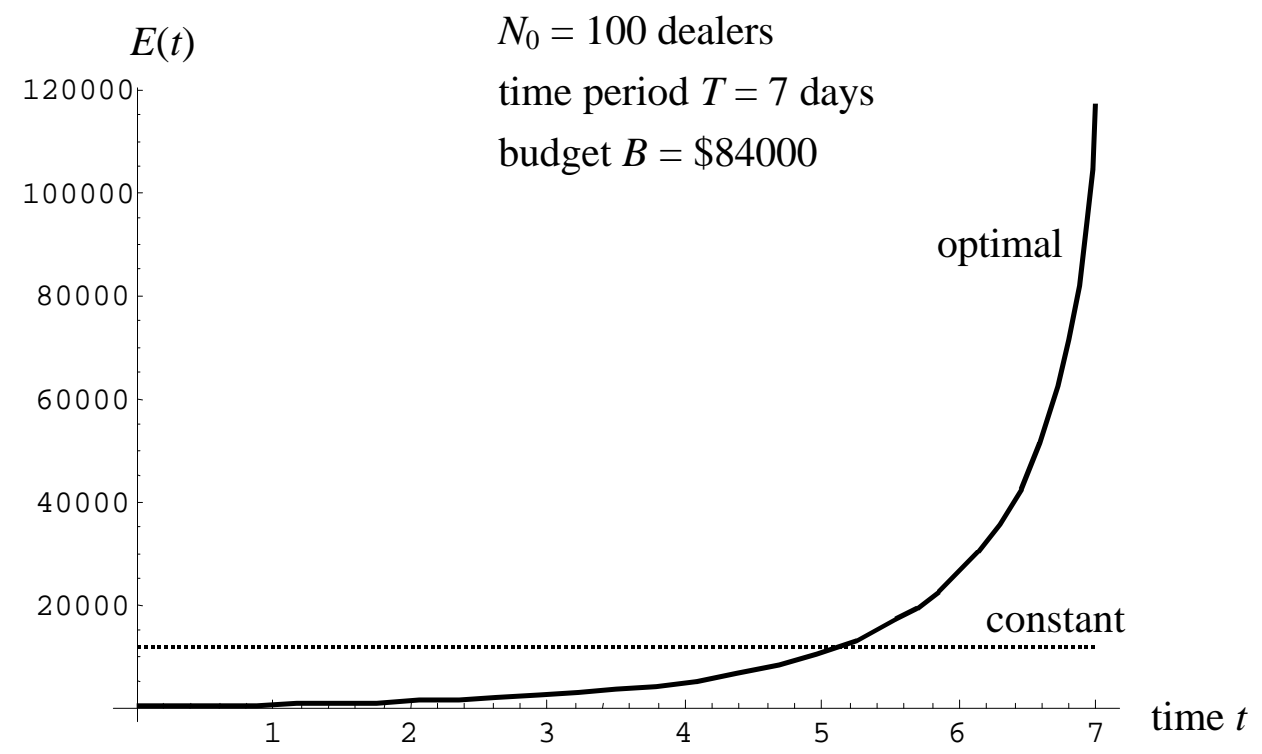

Figure 4. The optimal enforcement profile for a budget $B=\$ 84,000$ over the planning interval of $T=7$ days.

It is also instructive to plot how the number of dealers evolves over time as a reaction to the crackdown enforcement. Figure 5 shows that starting from $N(0)=100$ the dealer population decreases to $N(T)=8.5$. This optimal crackdown effect is compared with the constant enforcement $E=\$ 12,000$ spreading the $B=\$ 84,000$ equally over the 7 days (Figures 4 and 5). It is clear from the figures that while the higher enforcement effort up-front in case of constant enforcement yields very little benefit, the additional resources towards the end in the case of the optimal strategy indeed is very beneficial. This results in $N(7)=40$ for the case of constant enforcement versus $N(7)=8.5$ for the optimal strategy.

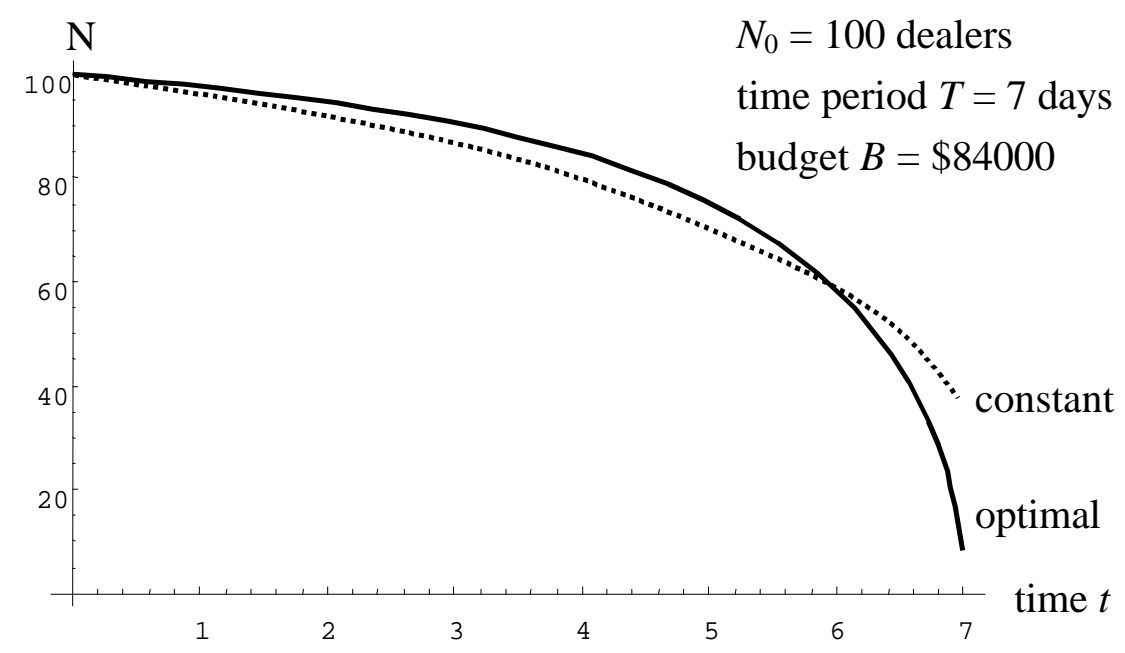

Figure 5. The time path of the dealers as they leave the market in case of optimal usage of budget $B=$ $\$ 84,000$ over the planning interval of $T=7$ days. 
Comparing the terminal dealer level $N(T)=8.5$ in Fig. 5 to $N_{\min } \approx 2.78$ and the terminal enforcement level $\mathrm{E}(\mathrm{T}) \approx 120000$ in Fig. 4 to $\hat{E} \approx 185000$, we see that our numerical example represents a part of trajectory I or II in Fig. 2 (never reaching $N_{\min }$ due to budget constraints).

\section{Sensitivity w.r.t. time and budget}

If the horizon time is increased from 1 week to 2 weeks, then spreading the same budget $B=$ $\$ 84,000$ optimally over $T=14$ days brings only a marginal reduction of final level, $N(T)$, compared to $T=7$; now we have $N(14)=7.9$ rather than $N(7)=8.5$.

In order to see the effect of available budget, $B$, and time, $T$, on the outcome $N(T)$ of the crackdown, we plot $N(T)$ for different values of $B$ and $T$ in Figure 6. From this figure it is easily obtained that increasing the budget sufficiently beyond $\$ 84000$ will lead to a market collapse, thus a situation where the number of dealers is reduced to zero ${ }^{6}$.

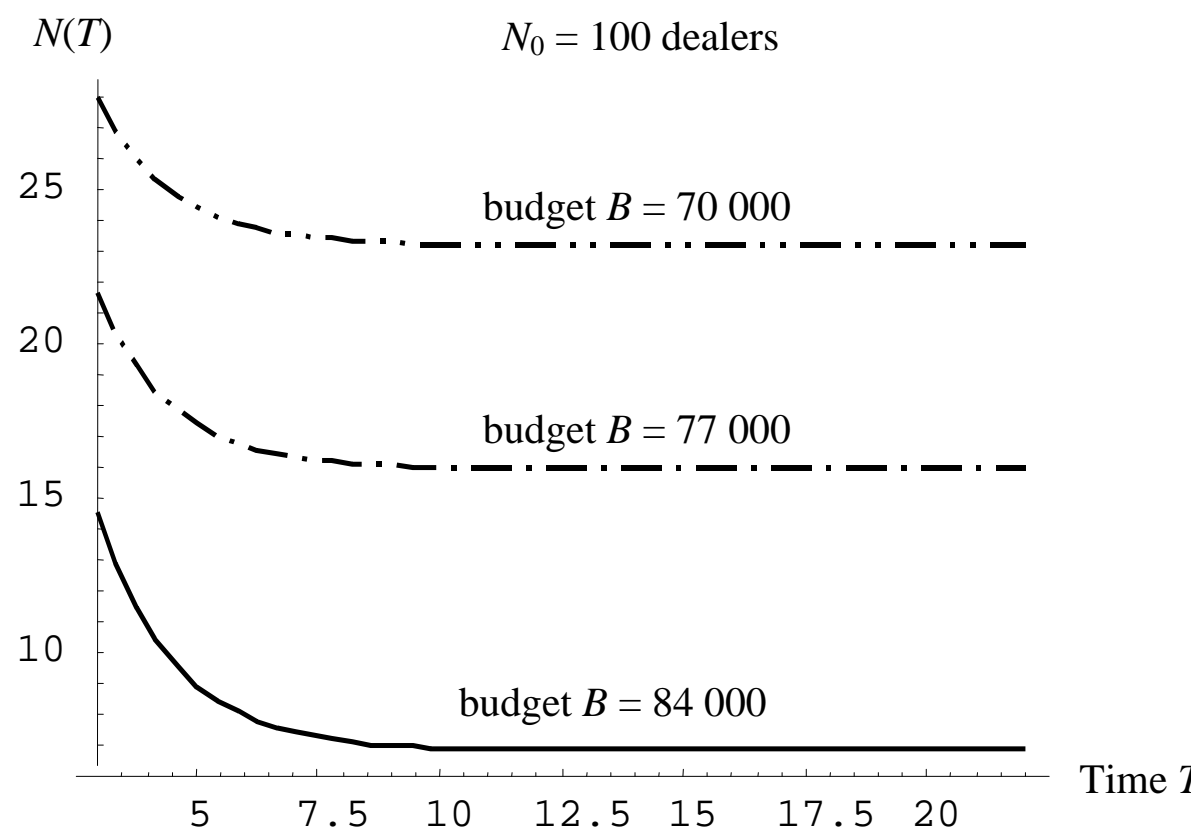

Figure 6. The outcome of the crackdown, $N(T)$, as a function of available time, $T$, for different values of budget $B$.

Figure 6 offers two clear policy implications relevant to this example:-

1. A reduction in budget to $\$ 70,000$ (compared to the earlier $\$ 84,000$ ) can have a severe detrimental effect and could jeopardize the success of the crackdown operation. In real terms, since the average personnel cost of a police officer for a 24-hour day is approximately $\$ 3,600$

\footnotetext{
${ }^{6}$ It turned out that it was very difficult to numerically compute a solution where such a market collapse actually occurs. The difficulty lies in the fact that for $E>0$ and $N \rightarrow 0$ (approaching the E-axis) it holds that $\dot{E} \rightarrow-\infty$ and $\dot{N} \rightarrow-\infty$.
} 
this reduction can be translated to two less officers or one less patrol car. This underscores the importance of each patrol car on the street during a crackdown operation.

2. Figure 6 also indicates that a crackdown length of one week seems reasonable. Less than one week leads to a significantly less impact in reducing the number of dealers. On the other hand, a time period greater than one week does not yield any additional significant benefits. This result is both important and consistent with the findings of Baveja et al. (1997) where they showed that a crackdown's value could be gauged soon - often during a week of a crackdown.

\subsection{Non-Risk-Seeking Dealers - Constant and Increasing Returns to Scale of Enforcement}

In case of decreasing returns to scale of enforcement, $\gamma<1$, the Hamiltonian was strictly concave in $E$. Therefore the optimal $E$ was continuous over time and was uniquely given by the solution of equation (11). This is no longer true in the case of constant returns to scale of enforcement, $\gamma=1$ (risk-neutral dealers), or increasing returns to scale of enforcement, $\gamma>1$ (riskaverse dealers), where the Hamiltonian is linear or convex in the control $E$. Here the optimal strategy could suggest use of infinite values of the enforcement, $E$, which are obviously unrealistic. Therefore, for $\gamma \geq 1$ it is necessary to have a realistic upper bound $\bar{E}$.

The decision problem now becomes

$$
\text { Minimize } e^{-r T} N(T)
$$

subject to:

$$
\begin{gathered}
\dot{N}=c_{1}\left[\pi \alpha N^{\beta-1}-\left(\frac{E}{N}\right)^{\gamma}-\omega_{0}\right], N(0)=N_{0}, \\
\dot{D}=E, \quad D(0)=0, \\
D(T) \leq B, \\
0 \leq E \leq \bar{E} .
\end{gathered}
$$

It will turn out that the cases $\gamma=1$ and $\gamma>1$ have the same optimal solutions. Thus, in order to avoid repeating arguments, we first analyze a linearized control problem that will then give the optimal solution for both cases.

\section{Linearization of the convex case}

In case of increasing returns to scale of enforcement, $\gamma>1$, we replace the function $h(E, N)=\frac{E^{\gamma}}{N^{\gamma}}$ by the function $\tilde{h}(E, N)=\frac{g}{N^{\gamma}} E$ with $g=\bar{E}^{\gamma-1}$. Thus $\tilde{h}(E, N)$ is linear in $E$. Furthermore it holds that: 


$$
\tilde{h}(0, N)=h(0, N) \text { and } \tilde{h}(\bar{E}, N)=h(\bar{E}, N)
$$

Due to (20) and the convexity of $h(E, N)$ in $E$, we obtain that within the allowed control region it holds that $\tilde{h}(E, N) \geq h(E, N)$, as depicted in Figure 7 .

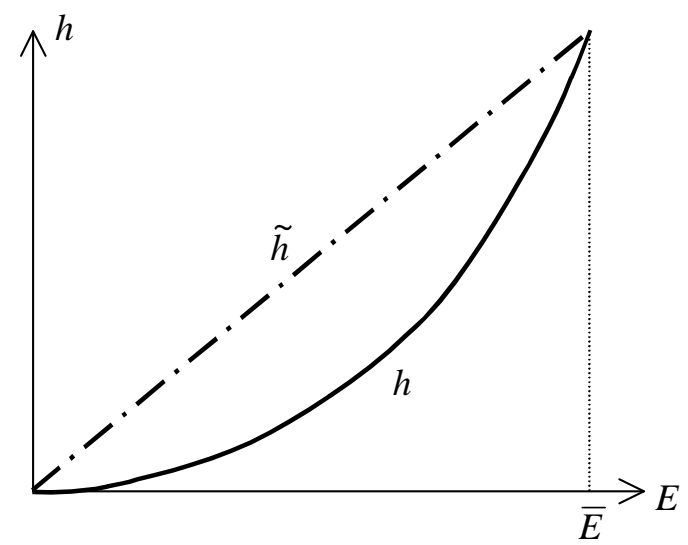

Figure 7. The original convex effectiveness of enforcement, $h$ and its linearized version, $\tilde{h}$ for fixed $N$.

We conclude that the value of the Hamiltonian corresponding to the model with the linear function represents an upper bound for the value of the Hamiltonian corresponding to the model with the convex function $h(E, N)$. Now the plan is to derive the optimal solution for the model with $\tilde{h}(E, N)$, and then approach this solution as much as possible while solving the original problem.

The model is:

$$
\operatorname{Max}-e^{-r T} N(T)
$$

subject to:

$$
\begin{gathered}
\dot{N}=c_{1}\left\{\pi \alpha N^{\beta-1}-g E N^{-\gamma}-w_{0}\right\}, \\
\dot{D}=E, \quad D(T) \leq B, \\
0 \leq E \leq \bar{E} .
\end{gathered}
$$

We now solve this linear model.

\section{Solution for the Linearized Model}

The Hamiltonian of this linear problem is:

$$
H=\lambda_{1} c_{1}\left\{\tau \alpha N^{\beta-1}-g E N^{-\gamma}-w_{0}\right\}+\lambda_{2} E
$$

Then, with the switching function

$$
\sigma=\frac{\partial H}{\partial E}=-\lambda_{1} c_{1} g N^{-\gamma}+\lambda_{2}
$$

the necessary conditions are the following: 
A Resource-Constrained Optimal Control Model for Crackdown on Illicit Drug Markets

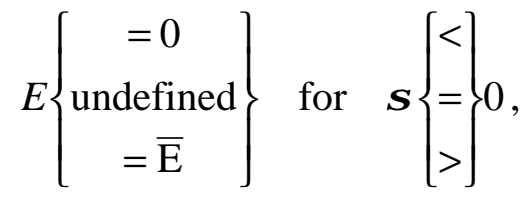

$$
\begin{aligned}
& \dot{\lambda}_{1}=r \lambda_{1}-\frac{\partial H}{\partial N}=\lambda_{1}\left\{r-c_{1}(\beta-1) \pi \alpha N^{\beta-2}-c_{1} \gamma g E N^{-\gamma-1}\right\}, \quad \lambda_{1}(T)=-1 \text {, } \\
& \dot{\lambda}_{2}=r \lambda_{2}, \quad \lambda_{2}(T) \leq 0 .
\end{aligned}
$$

We can identify 3 possible paths for positive $N$ depending on the value of $E$ relative to its boundary:

Path 1: maximum enforcement $E=\bar{E}$

Path 2: interior enforcement $0<E<\bar{E}$

Path 3: zero enforcement $E=0$

Using the necessary conditions we can prove the following Proposition:

\section{Proposition 3:}

a) a singular control (Path 2) can only happen for $N=N_{\min }$ and

$$
E=E^{*}=\frac{w_{0}}{g} N_{\min }^{\gamma}\left\{\frac{1-\beta}{\gamma+\beta-1}\right\}
$$

b) a switch to and from $E=E^{*}$ (Path 2) is only possible for $N=N_{\min }$

c) a switch from $E=O$ (Path 3) to $E=\bar{E}$ (Path 1) is only possible for $N \geq N_{\min }$

d) a switch from $E=\bar{E}$ (Path 1) to $E=O$ (Path 3) is only possible for $N \leq N_{\min }$

The proof is deferred to the Appendix. In the Appendix we also prove that it is not possible to connect Path 2 with any other path, so that it represents a hairline case which need not be considered any further. This is also in accordance with economic intuition since following Path 2 would mean staying at the level $N_{\min }$ which is not very reasonable because at this level the attractiveness of the market for new dealers is particularly high.

This yields the following proposition:

Proposition 3. The only possible sequences are

... Path $3 \rightarrow$ Path $1 \rightarrow$ Path $3 \rightarrow$ Path 1

... Path $1 \rightarrow$ Path $3 \rightarrow$ Path $1 \rightarrow$ Path 3

The necessary optimality conditions are satisfied by the following sequences consisting of only two paths: 


\section{Ramp-Up Sequence:}

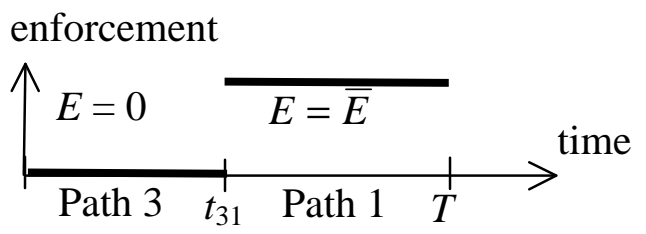

where $N \geq N_{\min }$ at the coupling time $t_{31}$. In most situations where $N_{0}=N_{\max }$ (i.e. the market is at a

high stable level of dealers), the time $t_{31}$ is chosen such that $\int_{t_{31}}^{T} \bar{E} d t=B$. In words, the crackdown operation will be delayed (until time $t_{31}$ ) to ensure that there are enough resources to sustain the maximum intensity of $E=\bar{E}$ until the end of the operation.

\section{Ramp-Down Sequence:}

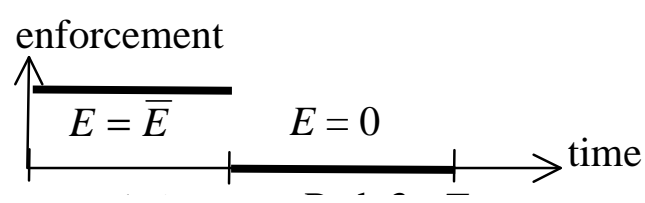

where $N \leq N_{\min }$ at the coupling time $t_{13}$. This can be considered a ramp down strategy, where the policy $E=\bar{E}$ is followed initially until the budget is used up.

Note that this sequence cannot be optimal if $N>N_{\min }$ at the coupling time, which happens if, for example, the budget is too small and/or $N_{0}$ is too large.

This policy will be optimal, if using $E=\bar{E}$ in a certain interval of length $t_{13}$ makes the market collapse, $N\left(t_{13}\right)=0$, and if the budget is large enough, $B \geq t_{13} \bar{E}$. After the market collapses at time $t_{13}$, enforcement $E$ can be reduced to zero and $N$ will not increase again. This result suggests putting all the resources up-front and with sufficient budget availability, can actually result in a market collapse by time $t_{13}$. Of course once the market collapses no crackdown enforcement will be required (recall that since $E$ is the crackdown enforcement in addition to the baseline level, a maintenance level would still be enforced).

From a practical standpoint it seems reasonable to expect a ramp-up sequence to be optimal when budget is insufficient to result in a significant collapse using the resources available. The argument for this would be as follows:- if a ramp-down sequence is used in such a low-budget scenario, after the switch to zero enforcement when the market is "still around", the market will grow and flourish suggesting that the ramp-up rather than ramp-down sequence may be beneficial. On the other hand, if sufficient enforcement resources are available for effecting a market collapse, ramp-down sequence seems reasonable. 
While it is an open question whether and in what situation sequences of 3 or more paths could be optimal, our conjecture is that such sequences will never be optimal.

\section{Solution for the Non-Risk-Seeking Dealers Model}

The results of the previous subsection immediately apply to the case of Risk-Neutral Dealers, $\gamma=1$, where we simply set $\gamma=g=1$.

Let us now turn to the case of increasing returns to scale of enforcement (Risk-Averse Dealers), $\gamma>1$, as was suggested by Baveja et al. (1997). As in the previous subsection, it is necessary to have an upper bound $\bar{E}$ on enforcement in order to prevent infinite values of $E$.

We note that for $\gamma>1$ the Hamiltonian

$$
H=\lambda_{1} c_{1}\left[\pi \alpha N^{\beta-1}-\left(\frac{E}{N}\right)^{\gamma}-\omega_{0}\right]+\lambda_{2} E
$$

is now strictly convex in the control, so that the Hamiltonian maximizing condition is similar to (27) for the linearized model:

$$
E=\left\{\begin{array}{l}
0 \\
\bar{E}
\end{array}\right\} \quad \text { if } \quad \lambda_{1} c_{1} \frac{1}{N^{\gamma}}\left\{\begin{array}{l}
> \\
<
\end{array}\right\} \lambda_{2} \bar{E}^{1-\gamma},
$$

while now interior controls $0<E<\bar{E}$ cannot occur and in the hairline case $E=0$ or $E=\bar{E}$ can be optimal. It is easy to show the following result:

Proposition 5. The optimal solution is the same as the optimal solution of the linearized model (21) - (24).

Proof. We know that the value of the objective function of this linear (maximization) model is an upper bound for the original model, since the effectiveness of enforcement is the same at the boundaries and is higher in the interior. Now, from the previous section we know, that the optimal solution of the linearized model will only consist of paths with boundary controls $E=0$ and $E=\bar{E}$, respectively. Thus the objective value of this solution for the original convex problem is the same as the objective value in the linearized model so that it is also optimal in the original convex problem. 


\section{Concluding Remarks}

Using optimal control theory, this paper analyzed the question of optimal drug crackdown enforcement policy, subject to a budget constraint. Based on the analysis of Caulkins' dynamic crackdown model, several insights were derived aimed at providing assistance to law enforcement managers in implementing drug crackdown operations. First, our analysis shows that in most realistic situations it is always optimal to use the entire enforcement budget. In other words, it rarely makes sense to leave unused resources, suggesting that a concentration of resources is indeed beneficial.

Second, combining Baveja et al.'s (1997) results of risk-averse dealer and those from our analysis of the risk-seeking dealer, it is reasonable to expect a crackdown operations' success to be gauged within a short period of time - e.g. a week. If crackdown operations will be successful, one can usually see it happening in a matter of days (and not months). This is consistent with the findings of actual crackdown operations - both successful and unsuccessful. For example, Buffalo, NY police department undertook a crackdown operation "STORM" in 1991-92, which eventually was called off after 14 months when success seemed unlikely. Our model argues that the lack of success in such cases is due to the resources being distributed over longer time duration instead of concentrating them for a shorter, more forceful operation. The model also questions the validity of waiting such a long time before recognizing the failure of the operation and suggests making a determination within a matter of week or so, which could save valuable resources in the long run.

Third, the paper shows that the optimal enforcement profile is a function of the risk aversion of a drug dealer. If the dealers are risk-seeking, the profit-making opportunity the latter part of the crackdown operation offers (due to fewer competitors) will make dealing attractive to them. This in turn will make reducing the size of the market harder as the operation progresses, suggesting use of an enforcement effort that increases with time. On the other hand, if the dealers are risk averse (or even risk neutral), the optimal strategy is one of using maximum resources as soon as the operation begins, until the market collapses ${ }^{7}$. One can rightly question the usefulness of these findings given that estimating the risk aversion of a drug dealer is difficult. Studying the economics of a drug market, Nell (1994) finds that law enforcement efforts catch the amateurs and the unorganized dealers improving market position of the more seasoned ones. Based on this finding, one possible characterization of a risk-averse dealer in Caulkins' model could be to consider them as seasoned and experienced. While this non-rigorous categorization of dealers may not be entirely accurate, it

\footnotetext{
${ }^{7}$ This assumes that enough budget is available. In case of insufficient budget availability, the optimal strategy is one of waiting and then using the maximum enforcement until the end of the crackdown period.
} 
may still be a good starting point, useful for estimating the risk profile of a dealer - a parameter the model clearly identifies as important in determining most efficient way of allocating crackdown enforcement resources. Finally, as mentioned earlier, a drug crackdown can never be successful in isolation and requires a post-crackdown effort as well. Typically, a crackdown operation is followed by a revitalization initiative aimed at normalizing the functioning of the area via improved street lighting, safe housing and efforts to relocate businesses and facilities to the neighborhood. Further, a maintenance enforcement level is needed to prevent a drug-market spring back especially in the case when crackdown budget was insufficient to result in a complete market collapse. It could be argued that the resources spent on this post-crackdown effort would be an increasing function of the number of dealers at the end of the crackdown phase, a quantity that our model minimized. Thus our model's results indirectly helps reduce the resources spent on follow-up operations. Our model did not explicitly incorporate the costs of follow-up operations since, often, these resources come from sources other than law-enforcement agencies e.g. city/mayor and federal empowerment funds. However, it is not difficult to see that any additional term in the objective function which is an increasing function of $N(T)$, will not alter the optimal enforcement profile the current model outputs.

The above-mentioned results, while significant, are clearly limited by the simplifying assumptions the model makes. The drug-dealing activity being modeled is illegal and consensual, making it difficult to measure directly, which makes certain assumptions necessary from a modeling standpoint. Despite these underlying limiting assumptions, we do believe that the model is a reasonable approximation of reality for several reasons. First, a profit-risk tradeoff framework which the model uses, and the accompanying underlying utility dynamics are a good estimate of the mental model of a money-driven activity. Further, the key characteristics of the dynamics in this model are that the actors (dealers in this case) flow in or out of the market in a self-interested manner, there is enforcement swamping, and the benefit of participating in the "market" is a decreasing function of the number of people in the market. These properties are so general that the basic model can be safely considered a good approximation of reality, and in fact, may also be applicable to purveyors of any black market commodity like, e.g., prostitution, numbers running, etc., or even burglary. Whether this modeling framework is indeed applicable to other illegal activities requires further investigation and is suggested as a direction for future research.

Another major limitation of the analysis presented here is that displacement of a dealer to other neighborhoods due to a crackdown operation has been essentially ignored. But as Baveja et al. (1997) argue displacement, while an important factor, cannot be used as a reason to disregard the model or argue against the effectiveness of crackdowns. Further, recent empirical study [NIJ 1996] 
reported little evidence of displacement of criminal activity to neighboring areas. Additionally, some researchers argue [Caulkins 1991] that since displacement could take the form of fewer negative externalities, a crackdown operation could still be beneficial despite occurrence of displacement. While these arguments do not justify not explicitly incorporating displacement in our model, it does offer some validity to the simplifying assumption.

With a growing effort among cities to reclaim crime-infested neighborhoods, the strategy of concentrating resources will probably be widely used in the future. However, many such operations that involve a concentration of resources may not be "crackdowns" in the restricted way this paper defines. Nevertheless, Caulkins' model and the accompanying analysis may prove to be a useful framework for defining a broader crackdown model - one which will have wider applicability to a variety of "clean-up" local enforcement operations that restrict/impede/arrest illegal activities/operations of individual criminals making decisions based on profit-risk tradeoffs.

\section{Appendix}

\subsection{Proof of Proposition 3}

Let us first compute the derivative of the switching function (26):

$$
\dot{\sigma}=-\dot{\lambda}_{1} c_{1} g N^{-\gamma}+\gamma \lambda_{1} c_{1} g N^{-\gamma-1} \dot{N}+\dot{\lambda}_{2}
$$

provided that this derivative exists. Otherwise this formula holds for the one sided derivatives because of (26) and because these derivatives exist for $N, \lambda_{1}$ and $\lambda_{2}$. Substitution of (22), (28) and (29) into (32) implies:

$$
\begin{aligned}
& \dot{\sigma}=-\lambda_{1} r c_{1} g N^{-\gamma}+\lambda_{1} c_{1}^{2} g(\beta-1) \pi \alpha N^{\beta-\gamma-2}+c_{1}^{2} \gamma g^{2} E N^{-2 \gamma-1} \lambda_{1}+\gamma \lambda_{1} c_{1}^{2} g \pi \alpha N^{\beta-\gamma-2} \\
& -\gamma \lambda_{1} c_{1}^{2} g^{2} E N^{-2 \gamma-1}-\gamma \lambda_{1} c_{1}^{2} g w_{0} N^{-\gamma-1}+r \lambda_{2}
\end{aligned}
$$

Rewriting gives:

$$
\dot{\sigma}=-\lambda_{1} r c_{1} g N^{-\gamma}+\lambda_{1} c_{1}^{2} g(\gamma+\beta-1) \pi \alpha N^{\beta-\gamma-2}-\gamma \lambda_{1} c_{1}^{2} g w_{0} N^{-\gamma-1}+r \lambda_{2}
$$

Now (26) can be used to simplify this further:

$$
\left.\dot{\sigma}=r \sigma+\lambda_{1} c_{1}^{2} g N^{-\gamma-1} \mid(\gamma+\beta-1) \pi \alpha N^{\beta-1}-\gamma w_{0}\right\rfloor
$$

Proof of part a) and $b$ ):

On a singular arc the following conditions hold:

$$
\sigma=0 \text { and therefore } \dot{\sigma}=0 \text {. }
$$

From (34) we can conclude that 


$$
N=N_{\min }=\left(\frac{\gamma+\beta-1}{\gamma}\right)^{\frac{1}{1-\beta}}\left(\frac{\pi \alpha}{\omega_{0}}\right)^{\frac{1}{1-\beta}}
$$

which is the same as (5) in the main paper. This in turn implies that

$$
E=E^{*}=\frac{\pi \alpha}{g} N_{\min }^{\beta+\gamma-1}-\frac{w_{0} N_{\min }^{\gamma}}{g}=N_{\min }^{\gamma}\left\{\frac{\gamma w_{0}}{(\gamma+\beta-1) g}-\frac{w_{0}}{g}\right\}
$$

which can be simplified to (30).

This completes the proof of a) and, by continuity of the state $N$, of b).

Proof of part c):

A switch from path $E=0$ to path $E=\bar{E}$ is only possible for $\sigma=0$ and $\dot{\sigma} \geq 0$, or, at least, $\sigma=0, \dot{\sigma}^{+} \geq 0$ and $\dot{\sigma}^{-} \geq 0$.

From (34) we can derive that $\sigma=0$ and $\dot{\sigma} \geq 0$ leads to

$$
(\gamma+\beta-1) \pi \alpha N^{\beta-1}-\gamma w_{0} \leq 0
$$

which is equivalent to

$$
N \geq N_{\min }=\left(\frac{\gamma+\beta-1}{\gamma}\right)^{\frac{1}{1-\beta}}\left(\frac{\pi \alpha}{\omega_{0}}\right)^{\frac{1}{1-\beta}}
$$

The Proof of part $d$ ) is completely analogous to that of part c).

\subsection{The Coupling Procedure}

The coupling procedure begins at the planning horizon and then works backwards in time. Since the transversality conditions $\lambda_{1}(T)=-1$ and $\lambda_{2}(T) \leq 0$ can hold on all paths [cf. the switching function (26)] all paths can be the final path. So we have three possibilities:

1. Path 1 with $E=\bar{E}$ is the final path

2. Path 2 with $E=E^{*}$ is the final path

3. Path 3 with $E=0$ is the final path

Path 1 is the final path: $E=\bar{E}$

At time $T$ it holds that

$$
\sigma=c_{1} g N^{-\gamma}+\lambda_{2} \geq 0 \text { with } \lambda_{2}(T) \leq 0
$$

which is possible. 


\section{Path $2 \rightarrow$ Path 1 is impossible:}

The continuity of the co-state variables and $N$ requires that:

$$
\sigma\left(t_{21}\right)=0, \quad \dot{\sigma}\left(t_{21}^{+}\right) \geq 0
$$

where $t_{21}$ is the point of time where Path 2 passes into Path 1. From Proposition $3 \mathrm{~b}$ we know that $N\left(t_{21}\right)=N_{\min }$ so that

$$
\left.\dot{\sigma}\left(t_{21}^{+}\right)=\lambda_{1} c_{1}^{2} g N^{-\gamma-1} \mid(\gamma+\beta-1) \pi \alpha N^{\beta-1}-\gamma w_{0}\right)=0 .
$$

Now it is interesting to compute the second derivative. From (34), i.e.,

$$
\dot{\sigma}=r \sigma+\lambda_{1} c_{1}^{2} g N^{-\gamma-1}\left[(\gamma+\beta-1) \pi \alpha N^{\beta-1}-\gamma w_{0}\right]
$$

we get the general expression

$$
\begin{aligned}
& \ddot{\sigma}=r \dot{\sigma}+\frac{d\left(\lambda_{1} c_{1}^{2} g N^{-\gamma-1}\right)}{d t}\left[(\gamma+\beta-1) \pi \alpha N^{\beta-1}-\gamma w_{0}\right] \\
& +\lambda_{1} c_{1}^{2} g N^{-\gamma-1}\left[(\gamma+\beta-1)(\beta-1) \pi \alpha N^{\beta-2} \dot{N}\right] .
\end{aligned}
$$

By $\dot{\sigma}=0$ and $N\left(t_{21}\right)=N_{\min }$ at the point of coupling, the first two terms vanish and (38) becomes

$$
\ddot{\sigma}\left(t_{21}^{+}\right)=-\lambda_{1} c_{1}^{2} g(\gamma+\beta-1)(1-\beta) \pi \alpha N^{\beta-\gamma-3} \dot{N}\left(t_{21}^{+}\right)<0 .
$$

Now $\sigma=0, \dot{\sigma}\left(t_{21}^{+}\right)=0$ and $\ddot{\sigma}\left(t_{21}^{+}\right)<0$ imply that $\sigma\left(t_{21}+\varepsilon\right)<0$ for all positive $\varepsilon$ small enough which is a contradiction to $E\left(t_{21}+\varepsilon\right)=\bar{E}$.

\section{Path $3 \rightarrow$ Path 1 is only possible for $\mathrm{N}>\mathrm{N}_{\min }$}

The continuity of the co-state variables and $N$ requires that:

$$
\sigma\left(t_{31}\right)=0, \quad \dot{\sigma}\left(t_{31}^{-}\right) \geq 0, \quad \dot{\sigma}\left(t_{31}^{+}\right) \geq 0 \text {. }
$$

From Proposition 3c) we know that $N\left(t_{31}\right) \geq N_{\min }$.

Assume for the moment that $N\left(t_{31}\right)=N_{\min }$. By $\sigma\left(t_{31}\right)=0$ and (34) this yields $\dot{\sigma}\left(t_{31}^{+}\right)=0$. As in (39) this implies $\ddot{\sigma}\left(t_{21}^{+}\right)<0$ which again gives the contradiction $\sigma\left(t_{21}+\varepsilon\right)<0$ and $E\left(t_{21}+\varepsilon\right)=\bar{E}$.

Thus only $N\left(t_{31}\right)>N_{\min }$ is possible at the switching time. Then, 


$$
\dot{\sigma}=r \sigma+\lambda_{1} c_{1}^{2} g N^{-\gamma-1}\left|(\gamma+\beta-1) \pi \alpha N^{\beta-1}-\gamma w_{0}\right|>0,
$$

which is in accordance with switching from $E=0$ to $E=\bar{E}$.

Proceeding in this way gives $\quad \ldots$ Path $3 \rightarrow$ Path $1 \rightarrow$ Path $3 \rightarrow$ Path 1

\section{Path 2 is the final path: $E=E^{*}$}

At time $T$ it holds that

$$
\sigma=c_{1} g N^{-\gamma}+\lambda_{2}=0 \text { with } \lambda_{2}(T) \leq 0,
$$

which is clearly possible.

\section{Switching to Path 2 is not possible}

This is because at $t_{12}$ or $t_{32}$ one would have $N\left(t_{x 2}\right)=N_{\min }$. By $\sigma\left(t_{x 2}\right)=0$ and (34) this yields $\dot{\sigma}\left(t_{x 2}^{-}\right)=0$. As in (39) this implies

$$
\ddot{\sigma}\left(t_{32}^{-}\right)=-\lambda_{1} c_{1}^{2} g(\gamma+\beta-1)(1-\beta) \pi \alpha N^{\beta-\gamma-3} \dot{N}\left(t_{32}^{-}\right)>0
$$

because of $E=0$ on path 3 and thus $\dot{N}\left(t_{32}^{-}\right)>0$. With $\sigma\left(t_{x 2}\right)=0$ and $\dot{\sigma}\left(t_{x 2}^{-}\right)=0$ this again gives the contradiction $\sigma\left(t_{32}-\varepsilon\right)>0$ against $\mathrm{E}\left(\mathrm{t}_{32}-\mathrm{e}\right)=0$.

On the other hand

because of $\quad-$ on path 1 and thus . With and this again gives the contradiction against

Notice that having Path 2 throughout is a hairline case ${ }^{8}$, since it can only occur when $N(0)=N_{\min }$ and $\quad$. Thus we need not consider Path 2 any further.

Thus we need not consider Path 2 any further.

\section{Path 3 is the final path: $E=0$}

At time it holds that

\footnotetext{
${ }^{8}$ Furthermore, it is economically clear that path 2 will not be used, since there - at - the market is particularly attractive for new dealers and thus keeping $N$ constant at this level is certainly expensive and not reasonable.
} 
which is possible.

\section{Path 2 Path 3 is impossible:}

The proof is the same as with Path 2 Path 1 above.

\section{Path $1 \quad$ Path 3 is only possible for $\mathrm{N}<\mathrm{N}_{\min }$}

The proof is the same as with Path 3 Path 1 above.

According to the previous arguments the resulting sequence will become $\quad$... Path $1 \quad$ Path 3 Path $1 \quad$ Path 3

\section{References:}

[1] Barnett, A. 1988. Drug Crackdowns and Crime Rates: A Comment on the Kleiman Report. In Street-Level Drug Enforcement: Examining the Issues, Marcia R. Chaiken (ed.). National Institute of Justice, Washington, D.C.

[2] Baveja, A., R. Batta, J. P. Caulkins and M. H. Karwan. 1997. When Haste Makes Sense: Cracking down on Street Market for Illicit Drugs. Socio-Econ. Plan. Sci 31 (4), 293-306.

[3] Baveja, A., R. Batta, J. P. Caulkins and M. H. Karwan. 1993. Modeling the Response of Illicit Drug Market to Local Enforcement. Socio-Econ. Plan. Sci 27 (2), 73-89.

[4] Caulkins, J. P. 1990. The Distribution and Consumption of Illicit Drugs: Some Mathematical Models and Their Policy Implications. Ph.D. Dissertation, Operations Research Center, Massachusetts Institute of Technology, Cambridge, Mass.

[5] Caulkins, J. P. 1993. Mathematical Models of Drug Markets and Drug Policy. Operations Research 41 (5), 848-863.

[6] Caulkins, J. P., R. C. Larson and T. F. Rich. 1993. Geography's Impact on the Success of Focused Local Drug Enforcement Operations. Socio-Econ. Plan. Sci 27 (1), 119-130.

[7] Caulkins, J. P. 1991. Thinking about Displacement in Drug Markets: Why Observing Change of Venue Isn't Enough. Journal of Drug Issues 22, 17-30.

[8] Feichtinger, G., Hartl, R.F., 1986, Optimale Kontrolle Ökonomischer Prozesse. De Gruyter, Berlin.

[9] Forero, J. 1990. Police Drug Squad Winning Support of Residents. The Buffalo News, August 26, p. B1 (continued on B6). 
[10] Hagga, J. G. and P. Reuter. 1990. The Limits of the Czar's Ukase: Drug Policy at the Local Level. N-3171-DPRC, Rand Corporation, Santa Monica, CA.

[11] Kennedy, D. 1990. Fighting the Drug Trade in Link Valley. C16-90-935.0, John F. Kennedy School of Government, Harvard University, MA.

[12] Kleiman, M. A. R. 1988. Crackdowns: The Effects of Intensive Enforcement on Retail Heroin Dealing. In Street-Level Drug Enforcement: Examining the Issues, M. R. Chaiken (ed.), National Institute of Justice, Washington, D.C.

[13] Kleiman, M. A. R. 1992. Against Excess: Drug Policy for Results. Basic Books, New York.

[14] Kort, P. M., G. Feichtinger, R. F. Hartl and J. L. Haunschmied. 1998. Optimal Enforcement Policies (Crackdown) on a Drug Market. Optimal Control Applications \& Methods 19, 169184.

[15] Naik, A. V., A. Baveja, R. Batta and J. P. Caulkins. 1996. Theory and Methodology: Scheduling Crackdowns on Illicit Drug Markets. European Journal of Operational Research 88, 231-250.

[16] National Institute of Justice. 1996. Policing Drug Hotspots. NIJ Research Preview, Washington D.C., January.

[17] Nell, E. J. 1994. The Dynamics of The Drug Market. Challenge 37, 13-21.

[18] Rydell, C.P., J. P. Caulkins and S.S.Everingham.1996. Enforcement or Treatment? Modeling The Relative Efficacy of Alternatives for Controlling Cocaine.Operations Research 44(5), 687-695.

[19] Sherman, L. W. 1990. Police Crackdowns: Initial and Residential Deterrence. In Crime and Justice: A Review of Research, Vol. 12, M. Tony and N. Morris (eds.). University of Chicago Press, Chicago.

[20] Worden, R. E., T. S. Bynum and J. Frank. 1992. Drugs and the Criminal Justice System. Sage, Beverley Hills, CA.

[21] Zimmer, L. 1987. Operation Pressure Point: The Disruption of Street-Level Drug Trade on New York's Lower East Side. Occasional Papers from The Center for Research in Crime and Justice, New York University School of Law, New York. 